\title{
Exploring Design Parameters for a 3D Head-Up Display
}

\author{
Nora Broy ${ }^{1,5}$, Simone Höckh ${ }^{2,3}$, Annette Frederiksen ${ }^{2}$, Michael Gilowski $^{2}$, Julian Eichhorn ${ }^{1}$, Felix Naser ${ }^{1}$, \\ Horst Jung ${ }^{2}$, Julia Niemann ${ }^{1}$, Martin Schell ${ }^{3}$, Albrecht Schmid ${ }^{4}$, Florian Alt ${ }^{5}$ \\ ${ }^{1}$ BMW Group - Forschung und Technik (Hanauerstr. 46, 80992 München, Germany) \\ ${ }^{2}$ Robert Bosch GmbH - Corporate Research (Robert-Bosch-Platz 1, 70839 Gerlingen-Schillerhöhe, Germany) \\ ${ }^{3}$ Technische Universität Berlin (Straße des 17. Juni 135, 10623 Berlin, Germany) \\ ${ }^{4}$ University of Stuttgart - VIS (Pfaffenwaldring 5a, 70569 Stuttgart, Germany) \\ ${ }^{5}$ University of Munich - Group for Media Informatics (Amalienstrasse 17, 80333 München, Germany)
}

\begin{abstract}
Today, head-up displays (HUDs) are commonly used in cars to show basic driving information in the visual field of the viewer. This allows information to be perceived in a quick and easy to understand manner. With advances in technology, HUDs will allow richer information to be conveyed to the driver by exploiting the third dimension. We envision a stereoscopic HUD for displaying content in 3D space. This requires an understanding of how parallaxes impact the user's performance and comfort, which is the focus of this work. In two user studies, involving 49 participants, we (a) gather insights into how projection distances and stereoscopic visualizations influence the comfort zone and (b) the depth judgment of the user. The results show that with larger projection distances both the comfort zone and the minimum comfortable viewing distance increase. Higher distances between the viewer and a real world object to be judged decrease the judgment accuracy.
\end{abstract}

\section{Categories and Subject Descriptors}

H.5.2 [Information Interfaces and Presentation]: User InterfacesScreen design (e.g., text, graphics, color)

\section{Keywords}

3D Displays; Human Factors; Automotive UIs; Head-Up Displays

\section{INTRODUCTION}

With the advent of head-up displays (HUDs) in the car, information can be presented within the visual field of the driver. Thus, the driver is able to keep the eyes on the road and simultaneously perceive information from these see-through displays. For example, the user does not need to switch his attention between the road and the instrument cluster for checking the current speed - hence HUDs can increase safety as well as comfort [11, 14]. Furthermore, augmented reality (AR) finds its way into automotive HUDs. AR HUDs enable direct mapping of virtual contents in the driving scene at their respective positions [14]. Current research considers monoscopic solutions by using a projection plane at a distance of

Permission to make digital or hard copies of all or part of this work for personal or classroom use is granted without fee provided that copies are not made or distributed for profit or commercial advantage and that copies bear this notice and the full citation on the first page. Copyrights for components of this work owned by others than the author(s) must be honored. Abstracting with credit is permitted. To copy otherwise, or republish, to post on servers or to redistribute to lists, requires prior specific permission and/or a fee. Request permissions from Permissions@acm.org.

PerDis'14, June 03 - 04 2014, Copenhagen, Denmark

Copyright is held by the owner/author(s). Publication rights licensed to ACM. ACM 978-1-4503-2952-1/14/06 ... \$15.00.

http://dx.doi.org/10.1145/2611009.2611011 more than $10 \mathrm{~m}$ in front of the driver. Due to this distance, the required focus changes to alternately perceive the HUD content and the driving scene in the real world is relatively small. Yet, to position the content in space, AR HUDs rely on depth cues such as relative size.

We envision a stereoscopic HUD (stereo HUD), as used in numerous consumer devices, to tackle this issue. A stereo HUD uses binocular disparity as depth cue to generate 3D vision. It embeds driving-relevant information into the driving scene using the third dimension: A stereo HUD enables content to be perceived at variable distances as well as presenting objects in various depth layers simultaneously. For example, a navigation arrow could be shown directly at the respective intersection while driving parameters, such as speed, could be perceived in front of the car.

Today, research on stereo HUDs is scarce. However, as prior work on the use of stereoscopic 3D displays in the car suggests, the use of extreme parallaxes may cause discomfort and, hence, may have a negative influence on the driver [1]. Furthermore, to present image content so that the driver perceives it at the respective location matching the real world the accuracy of depth judgment in a stereoscopic see-through display has to be explored.

In this work, we present two studies that investigate both the parallax range allowing for comfortable vision as well as the effect of object placement with regard to the depth judgment accuracy. The contribution of this work is twofold. First, we provide a methodology for assessing the depth range in which image content can be placed to still allow for comfortable vision using different projection distances of a see-through 3D display. Second, our two studies provide a data set that influences the technical as well as the content design of a 3D see-trough display system dependent on the envisioned application area.

\section{RELATED WORK}

Our work draws from prior research in the area of 3D displays and head-up displays. In the following, we introduce related work, discuss what we learn from it, and point out major differences.

\subsection{D Displays}

In general, (stereoscopic) 3D displays use binocular disparity as depth cue, thus presenting different images for each of the viewer's eyes. However, 3D displays can evoke discomfort and fatigue [6]. For a given distance between viewer and projection plane there is a corresponding depth region in which the image content can be displayed for a comfortable 3D experience. This region is called the comfort zone [9]. One of the factors influencing the comfort zone is the vergence-accommodation conflict [4, 9, 13]. Using a stereoscopic display, the eyes focus on the screen while converging 
on the respective object displayed in front or behind the screen. If the difference between the depth information accommodation and convergence is too large, discomfort will arise and, eventually, the fusion of the single images will not be possible any more.

There are several studies that investigated the comfort zone [1, $5,9,15]$ of 3D displays. However, these studies investigate small viewing distances and do not apply see-through displays. In contrast, we focus on larger viewing distances of up to $15 \mathrm{~m}$.

Depth perception can be assessed through measuring the accuracy of depth estimations. Swan II et al. provide a good overview of measurements for depth estimations [10]. They also provide a comprehensive study of judging depth positions of real world objects via virtual objects presented by an optical see-through display. However, they focus on depth judgments for a single viewing distance. In this work, we investigate the accuracy of depth judgments for various distances between projection plane and viewer.

\subsection{Head Up Displays}

Many automotive manufacturers apply HUDs in their cars, particularly in upper class vehicles [11]. Using a traditional instrument cluster, the driver is forced to permanently perform stressful changes of focus to look at the display and the driving scene in turns. The larger projection distance of a HUD decreases this change of focus and thereby increases safety and comfort [11, 14]. For example, Milicic and Lindberg [7] show that a HUD increases secondary task performance as well as driving performance, compared to a head-down display (i.e., a classical instrument cluster).

An automotive HUD utilizes the windshield as a partly reflecting mirror. Nowadays, automotive HUDs typically present a virtual image approximately $2 \mathrm{~m}$ in front of the driver [7]. Ott et al. show the functionality of a HUD system [8]. Currently, AR HUDs are developed that allow displaying information at larger projection distances of more than $10 \mathrm{~m}$ due to their particular optical design. For example, Tönnis et al. use a distance of $13 \mathrm{~m}$ to investigate arrowbased route guidance systems for an AR HUD [12].

\section{STEREOSCOPIC HEAD UP DISPLAY}

We see large potential in augmenting the real driving scene with virtual content by means of a stereo HUD. This allows us to show content at a variable distance from the driver (in the following referred to as virtual image distance or VID) although the projection distance (virtual screen distance or VSD) is fixed. Additionally, a stereo HUD can display content at different VIDs simultaneously.

\subsection{Motivation}

Monoscopic solutions only allow for two dimensionally augmenting the real world. We believe that augmenting it via a stereo HUD clarifies the virtual augmentation because of a close link between the displayed content and the surrounding driving scene: The VID of a virtual object can be adapted to the distance of the respective real world reference. Hence, a navigation arrow can appear at the same distance where the next turnoff is located.

Another benefit is that different depth planes can be addressed simultaneously. In this way, the usability of the interface can be improved by semantically structuring the displayed information via depth [2]. For example, an intuitive spatial arrangement could include showing navigation information further away from the driver than speed. Additionally, warnings that concern the vehicle, such as low fuel level or an open door, can be displayed closer to the driver. They could even move towards the driver when the information becomes more relevant. Regarding the increasing amount of information a driver has to operate while driving (e.g., concerning safety and comfort), this aspect could become even more pronounced.
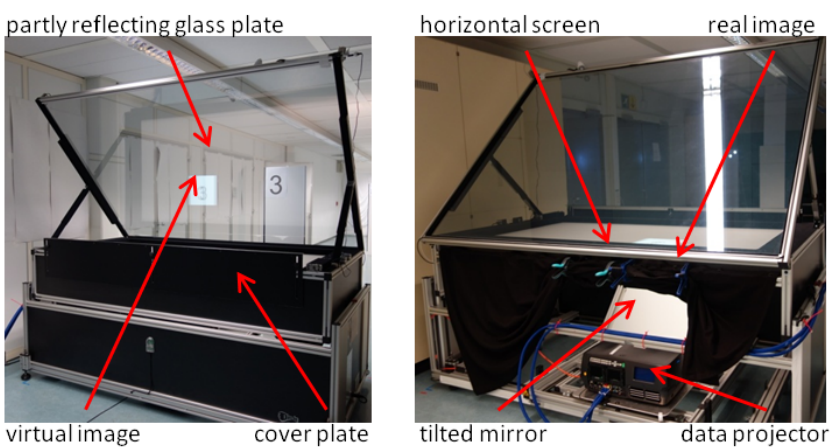

Figure 1: Laboratory device to perform 3D see-through display studies: front side (left) and back side (right) of the emulator.

The VSD is determined by the optical design of the system. It has to be chosen depending on the aspired VID range to display the virtual image within the user's comfort zone. In our first study, we examine the relation between VSD and comfortable VID range. We use an appropriate testing device to assess parameters that are crucial for designing the optical system and the content.

\subsection{Apparatus}

The centerpiece of the experimental setup is a 3D emulator (Figure 1). It allows virtual stereoscopic images with variable VSDs and VIDs to be created. As projection unit, we use a Projectiondesign F35 AS3D projector capable of presenting 3D images. It projects on a horizontal screen with a height of $95 \mathrm{~cm}$ via a tilted mirror inclined by $45^{\circ}$. We mount a glass plate with a visual reflectance of $40 \%$ and a size of $2,2 \mathrm{~m} \times 1,6 \mathrm{~m}$ diagonally above the screen. Hence, the real image on the screen can be observed as a virtual image, superimposed with the surroundings.

The system uses shutter technology and works with a frequency of $60 \mathrm{~Hz}$ per eye, resulting in a total frequency of $120 \mathrm{~Hz}$. We apply an adapted version of the software Workbench $3 \mathrm{D}^{1}$ in which we can set parameters like eye distance, VSD, and pixel size. Subsequently, the displayed virtual objects can be varied with regard to their VID, position, and size. The user can adapt the parallax of virtual objects by means of a game controller (Speedlink XEOX Pro Analog Gamepad). Binocular disparity is the only varying depth cue. Thus, the size of a virtual object is constant while its depth position changes. The look down angle of $0^{\circ}$ is kept constant. To realize different VSDs, the emulator is mounted flexibly. A corridor of $22.4 \mathrm{~m}$ length allows us to investigate a huge VSD range. To provide the desired surrounding conditions, we adapt the illumination of the room and the homogeneity of the wall color.

\section{COMFORT ZONE EXPLORATION}

Since excessive parallaxes have a strong impact on the viewing comfort (cf. Section 2.1), it is essential to determine a proper image distance range for a given screen distance. Suggested limits for parallaxes strongly vary among former investigations [5]. Moreover, Williams and Parrish [15] showed that increasing the screen distance expands the limits of the comfort zone. They investigated distances up to $1.4 \mathrm{~m}$. However, we are interested in screen distances between 2 and $15 \mathrm{~m}$.

In this study, we investigate five different VSDs within this depth range and the respective comfort zones. We aim to find out the participants' individual comfort zones by asking them to alter the depth position of the virtual objects to the borders of their comfort

\footnotetext{
${ }^{1}$ http: / / www . workbench3d.de/
} 


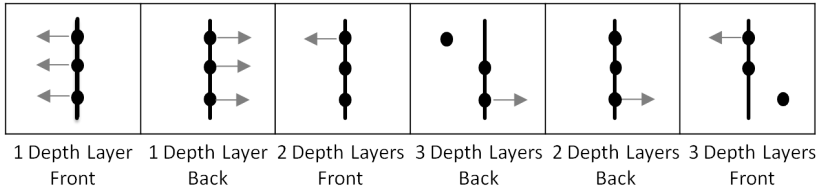

Figure 2: Sequence of the direction and the number of depth layers combinations for one of the six groups.

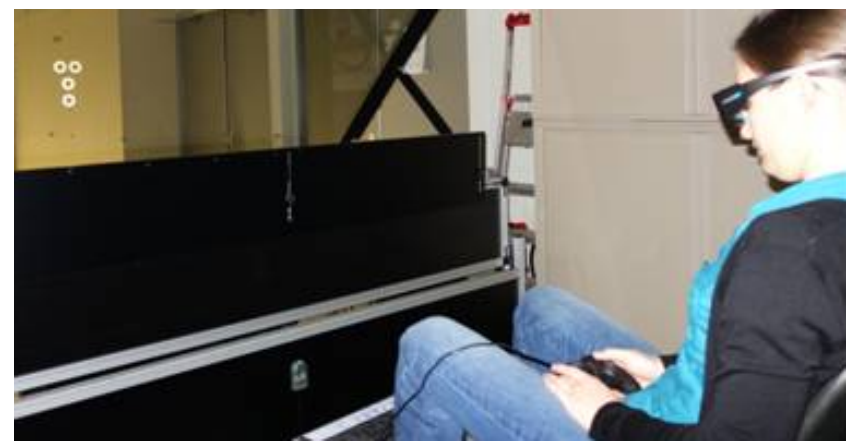

Figure 3: Participant adjusting the topmost ring to the limit of her personal comfort zone.

zones. This approach is similar to Broy et al. [1]. In this way, we identify the maximum and minimum depth positon relative to the respective VSD at which the viewer is still able to fuse content in a comfortable way. As virtual content elements we use vertically aligned rings (cf. Figures 2 and 3), with an angular size of approximately $0.6^{\circ}$. The participants use a game pad to vary the depth position of the virtual rings.

\subsection{Study Design}

The study follows a repeated measures design, exposing all participants to all combinations of the following independent variables.

VSD: As we are interested in the effect of the VSD on the resulting comfort zone we investigate five different VSDs $(2 \mathrm{~m}, 3 \mathrm{~m}$, $5 \mathrm{~m}, 8 \mathrm{~m}$, and $15 \mathrm{~m}$ ).

Direction: Starting from the respective VSD, the participants can determine their individual limit of the comfort zone to the front (negative parallax) and to the back (positive parallax).

Number of depth layers: We explore the impact of the number of virtual depth layers on the comfort zone. Therefore, we distinguish three cases. Either, the depth position of the three virtual rings is changed simultaneously (just one virtual depth layer is presented). Or the depth position of just one ring is adjusted while the other two rings stay on the same layer, (two depth layers). Or one ring is altered while the other two rings occupy different depth layers (three depth layers).

Since the adjustment of different VSDs requires to rearrange the emulator, the study is divided into five parts - one for each VSD condition. To avoid sequence effects we present the different VSDs in random order. We counterbalance the combinations of the direction and the number of depth layers using a latin square. This results in $2 * 3=6$ combinations. Thus, we divide our test sample in six different groups that experience the respective sequence of the direction and the number of depth layers combinations. Figure 2 shows the sequence for one of the six groups. For one group the respective sequence is obtained for each VSD part. As dependent variable we measure the absolute adjusted parallax value.

\begin{tabular}{lccc}
\hline Factor & Level & Mean & SD \\
\hline \multirow{3}{*}{ Depth layers } & 1 & 147.595 & 87.918 \\
& 2 & 84.764 & 72.826 \\
& 3 & 81.085 & 74.980 \\
\hline \multirow{2}{*}{ Direction } & front & 161.740 & 140.263 \\
& back & 47.222 & 14.445 \\
\hline \multirow{3}{*}{ VSD } & $2 \mathrm{~m}$ & 50.757 & 31.850 \\
& $3 \mathrm{~m}$ & 65.902 & 40.242 \\
& $5 \mathrm{~m}$ & 93.649 & 76.066 \\
& $8 \mathrm{~m}$ & 122.120 & 96.240 \\
& $15 \mathrm{~m}$ & 189.978 & 147.426 \\
\hline
\end{tabular}

Table 1: Descriptive statistics of the absolute parallax values in mm for the main effects.

\subsection{Procedure}

We started each test session by asking about demographic data and former experiences with stereoscopic 3D. We measured the interpupillary distance of each participant with a pupillometer to calculate the respective VIDs depending on the VSD, the interocluar distance, and the adjusted parallax value. Then we assessed general (corrected) eyesight, using a Snellen test. Participants used both eyes simultaneously. Furthermore, we used Random Dot Stereograms (RDSs) to test their ability to perceive stereoscopic 3D.

As the participants had successfully passed these tests, they tried out the setup to get used to the system and the task. During this phase, we asked them to move the virtual object out of their comfort zone intentionally, so that the corresponding feeling could be experienced and recognized during the actual test. After that, the five VSD test condition blocks were presented in accordance to the study design. For each test condition the participants adjusted their individual comfort zone limits. During the adjustment, participants were asked to change their focus momentarily to a different point in the room. When the participants had decided on the limit of their personal comfort zone, they informed the experimenter who logged the value. In addition to the main task, we conducted a semistructured interview to find out about the participant's subjective experience. We asked questions about the effort and discomfort the participant felt and about the subjective degree of task difficulty.

\subsection{Results}

In total, the results of 24 subjects (female: 5, male: 19) aged 27 to $74(M=46, S D=11)$ were evaluated. All 24 subjects had normal or corrected to normal vision and passed the RDS test.

Figure 4 visualizes the descriptive statistics for the rated limits of parallaxes that provide comfortable viewing. Descriptive statistics for the main effects are outlined in Table 1. Since a KolmogorovSmirnov test shows that our data is not normally distributed $(p<$ $.05)$ we use non parametric tests for the statistical analysis. We use Friedman tests for the main effects and Wilcoxon tests with Bonferroni corrected significance levels for pairwise comparisons.

Comparing the different VSD levels with a Friedman test shows statistical differences, $X^{2}(4)=77.10, p<.001$. Wilcoxon tests show significant differences for all pairwise comparisons, $p<.005$, except for 3 vs. $5 \mathrm{~m}, p=.006$. As Table 1 and Figure 4 show the parallax limits of the comfort zone increase for higher VSDs.

The number of depth layers has a significant influence on the parallax limits, as a Friedman test confirms, $X^{2}(2)=37.33, p<$ .001 . Wilcoxon tests show that the participants can handle higher parallaxes for one vs. two layers, $Z=-4.286, p<.001, r=$ -.619 , and one vs. three layers, $Z=-4.286, p<.001, r=$ 


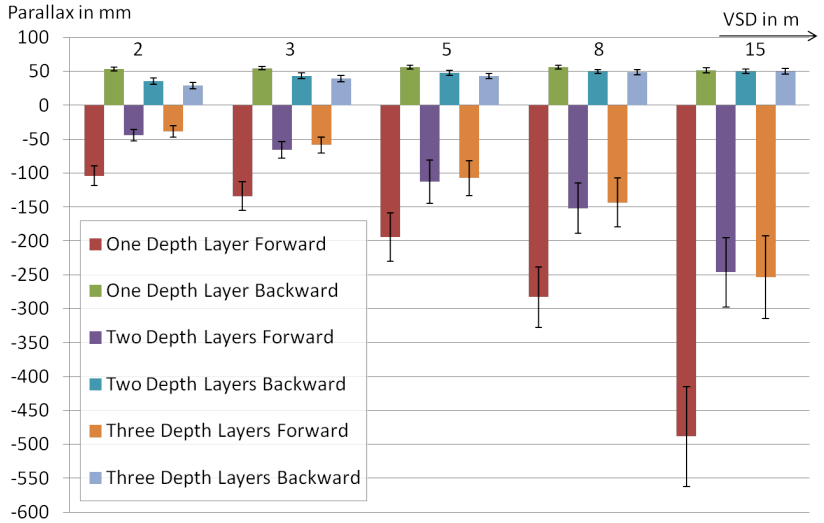

Figure 4: Means and standard errors for the parallaxes in $\mathbf{m m}$ rated as the limits for a comfort zone.

-.619 . However, there are no significant differences between presenting two vs. three layers, $Z=-1.571, p=.116, r=-.227$.

Finally, the absolute parallax limits for negative parallaxes (to the front) are significantly higher than for positive parallaxes (to the back), $Z=-4.200, p<.001, r=-.606$.

The interviews revealed that most participants perceived the $3 \mathrm{D}$ effect as comfortable (92\%) and appreciate the 3D effect for an automotive HUD (92\%). $33 \%$ of the participants positively mentioned a VSD of $5 \mathrm{~m}$, while there are just $29 \%$ for $8 \mathrm{~m}, 25 \%$ for $15 \mathrm{~m}$, and $4 \%$ for $3 \mathrm{~m}$ and none for $2 \mathrm{~m} .29 \%$ criticized the $2 \mathrm{~m}$ VSD. Only $8 \%$ negatively commented on the $15 \mathrm{~m} \mathrm{VSD,} 4 \%$ on the $3 \mathrm{~m}$ VSD. The VSDs of 5 and $8 \mathrm{~m}$ got no negative comments.

\subsection{Discussion}

Our results show that higher VSDs allow larger parallaxes to be presented. In accordance, Shibata et al. [9] show that there is a higher overall fatigue for near viewing distances compared to far viewing distances. They did not use a see-through display but viewing distances of up to $10 \mathrm{~m}$. In comparison, our study addresses several VIDs above $1 \mathrm{~m}$ and we aim to find values that define the comfort zone for the tested VSDs of a see-through display. In accordance to the approach of Broy et al. [1], we use the medians and the quartiles of the data to define a comfort zone. The resulting data set is available for public use from our website ${ }^{2}$.

For each VSD * direction condition we found comfort zone limits aggregated for two and three depth layers. We aggregate the data due to the outcome that there are no statistical significances between two and three depth layers. Broy et al. [1] compared the number of depth layers as well and also show that one depth layer provides larger comfort zones than two depth layers. Yet, their study does not investigate more than two layers. Our results yield no significant differences between two and three depth layers. This indicates that it is possible to assess the comfort zone by just distinguishing between one and multiple depth layers. How a multiple depth layer condition may look like depends on the application.

Since our results indicate that if two depth layers are occupied, the use of additional depth layers will not directly decrease the comfort zone. For the case of developing a stereo HUD, we identified a VSD between $5 \mathrm{~m}$ and $8 \mathrm{~m}$ as promising since it allows content from approximately $3 \mathrm{~m}$ up to $20 \mathrm{~m}$ in front of the driver to be shown. Figure 5 shows the difference between one and more depth layers for the comfort zone pertaining a VSD of $5 \mathrm{~m}$. We calculated the VIDs in respect to the measured interocular distance. The used

\footnotetext{
${ }^{2}$ Data set: http://hcilab.org/automotive/3dhud/
}

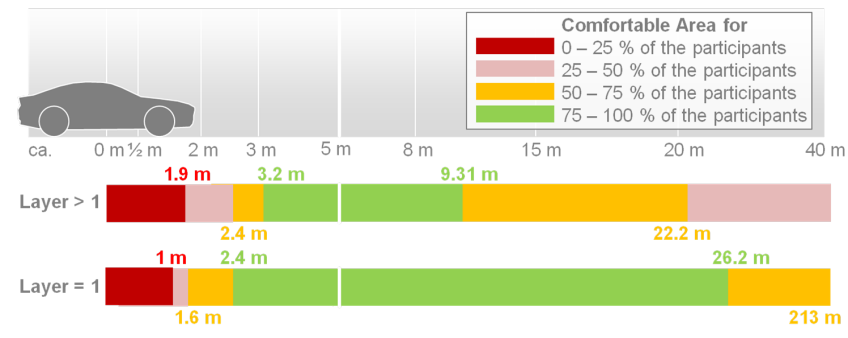

Figure 5: Comfort zone limits represented as distances from the VSD, which is $\mathbf{5} \mathbf{m}$ in this case.

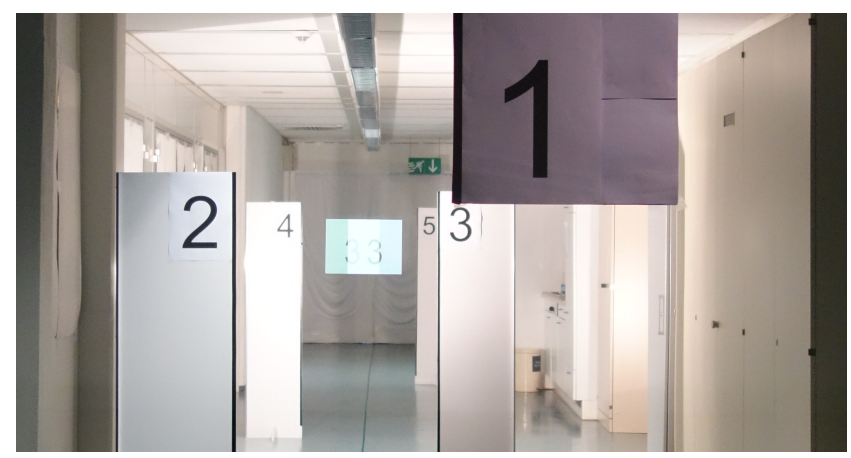

Figure 6: Setup of the depth judgment study: participant's view with real world shields and a numbered virtual square.

pupillometer has an accuracy of measurement of $0.5 \mathrm{~mm}$, and hence a potential influence on the calculated VSDs. However, we assume that possible measurement errors are normally distributed and have no significant influence on our results.

Shibata et al. [9] show that negative parallaxes are less comfortable for small viewing distances and positive parallaxes are less comfortable for far viewing distances. In accordance, our study reveals that still comfortable parallaxes are higher for negative than for positive parallaxes as we investigate high VIDs with VSDs beyond $2 \mathrm{~m}$. Still, we prevented our participants from diverging eye movements through restricting positive parallaxes.

\section{DEPTH JUDGMENT ACCURACY}

The second study investigates the accuracy of judging the depth positions of real world objects via a virtual object. We use a similar setup as in our first study. The emulator which is described in Section 3.2 shows a square of approximately 3.4 to $3.9^{\circ}$ angular size with a number on it. The test environment is equipped with white shields with a black marked edge. They are placed on pre-defined positions and carry black numbers (cf. Figure 6).

We apply a perceptual matching task as described by Swan et al. [10]: The participants' task is to adjust the depth of the virtual object to the depth of the real world shield with the respective number. The participants use a game pad to manipulate the position of the numbered square in discrete steps of $5 \mathrm{~cm}$. If the participants perceive the virtual object at the same depth as the respective shield, they confirm the depth by pressing a button on the game pad.

\subsection{Study Design}

The study is designed as a repeated measure experiment with the following independent variables.

VSD: In accordance to our initial study, we test the depth judgment accuracy for different VSDs at $3 \mathrm{~m}, 5 \mathrm{~m}$, and $8 \mathrm{~m}$. 


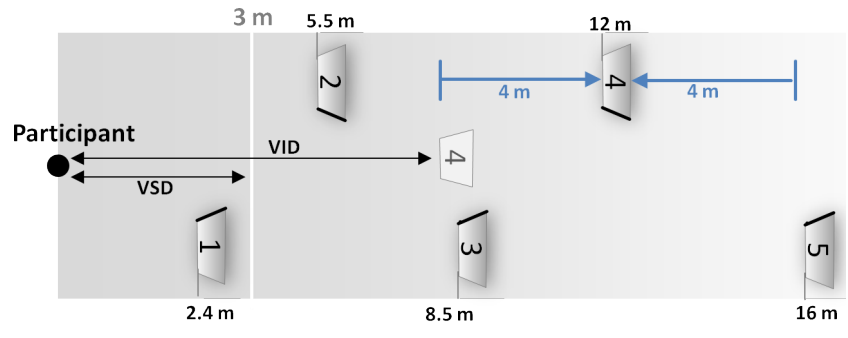

Figure 7: Top view of the study setup for a VSD of $3 \mathrm{~m}$. The participants adjusted the virtual object to its corresponding shield (in this case shield 4). The initial position of the virtual object is once $4 \mathrm{~m}$ in front and once $4 \mathrm{~m}$ behind the respective shield.

Shield Position: The participants judge the depth of five different shield positions for each VSD. The shields have a distance from the viewer of $2.4 \mathrm{~m}, 5.5 \mathrm{~m}, 8.5 \mathrm{~m}, 12 \mathrm{~m}$, and $16 \mathrm{~m}$.

One test session is divided into three parts, one for each VSD level. We counterbalance the order of the VSDs by dividing our test sample in $3 !=6$ groups. One participant judges the depth position four times for each VSD and shield combination. Thereby, the initial position of the virtual object is $4 \mathrm{~m}$ in front or behind the shield which has to be judged (cf. Figure 7) except for the first two shields. Here, the virtual image appears at a VID of $2 \mathrm{~m}$ when we start in front of the shield. Each shield is approached twice from the front and twice from the back for each VSD. This results in $4 * 5=20$ depth judgments for each VSD level. The sequence of the 20 tasks for one VSD part is randomized. In total, one participant provides $20 * 3=60$ depth estimations. As dependent variable we measure the absolute offset between the adjusted VID position of the virtual square and the respective real world shield. In the remainder, we refer to this value as the absolute judgment error.

\subsection{Procedure}

The initial assessment of demographic data, the measurement of the participant's eye distance, and the preceding tests were performed analog to the first study. Again, the participant acquainted with the system and the task during a training run. After that, two test runs for each VSD occurred. Each run included the approach of all of the five shields from both directions in respect to the study design. A schematic overview of the setup is shown in Figure 7. Again, the setup had to be rearranged to realize the different VSDs. Different additional light sources were used in order to illuminate the shields and their numbers sufficiently. We conducted an accompanying interview similar to the one described above for study 1 .

\subsection{Results}

In total, the results of 25 participants (female: 7, male: 18) aged from 34 to $65(M=47, S D=10)$ were evaluated. All 25 participants had normal or corrected to normal vision and passed the RDS test.

Figure 8 shows the mean and standard errors of the absolute judgment error for the test conditions. Table 2 outlines the descriptive statistics for the main effects concerning VSD and shield position. A Kolmogorov-Smirnov test shows that the data is not normally distributed. As in study 1, we used Friedman and Wilcoxon tests with Bonferroni corrections for further statistical analysis.

Comparing the shield positions a Friedman test shows that the accuracy of judging various real distances differs significantly, $X^{2}(4)=$ 70.237, $p<.001$. Bonferroni corrected Wilcoxon tests show significant differences for all pairwise comparisons, $p<.005$, except for shield 1 vs. $2, p=.119$ as well as for shield 1 vs. $3, p=.016$.

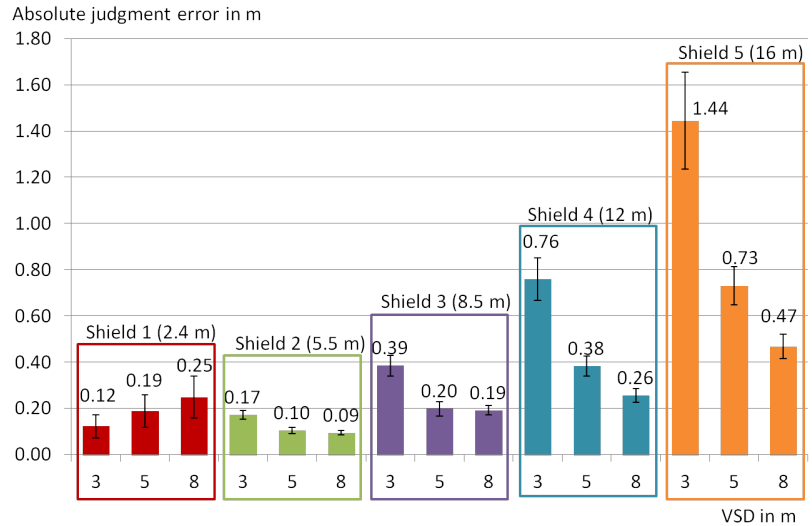

Figure 8: Means and standard errors for the errors of the absolute depth judgment.

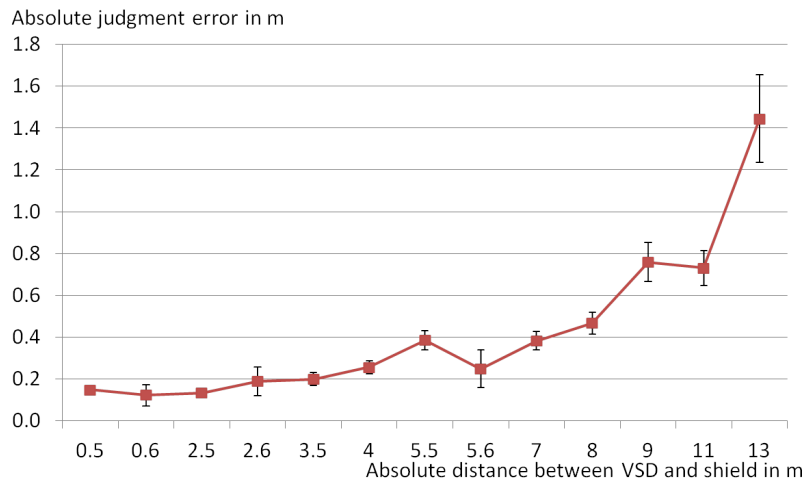

Figure 9: Means and standard errors for the absolute depth judgment error regarding distance between shield and VSD.

The different VSD levels provide significantly different accuracies for judging the shield positions as a Friedman test shows, $X^{2}(2)=22.92, p<.001$. Wilcoxon tests with a bonferroni corrected significance level reveal significant differences for all pairwise comparisons, $p<.005$. Figure 8 , which directly pictures the absolute judgment error, implies that the accuracy of the judgment decreases with increasing VSD value for shield 1 . For all other shields the effect occurs vice versa. This leads to the assumption that an increasing distance between shield and VSD impairs the accuracy of the depth judgment. Figure 9, again depicting the absolute judgment error, represents the accuracy of the depth judgment based on the distance between the shields and VSDs. It shows that a growing distance between VSD and shield leads to a decrease of the depth judgment accuracy. A Friedman test affirms this finding, $X^{2}(12)=184.110, p<.001$.

\begin{tabular}{lccc}
\hline Factor & Level & Mean & SD \\
\hline \multirow{3}{*}{ VSD } & $3 \mathrm{~m}$ & 0.577 & 0.337 \\
& $5 \mathrm{~m}$ & 0.321 & 0.145 \\
& $8 \mathrm{~m}$ & 0.252 & 0.138 \\
\hline \multirow{3}{*}{ Shield } & 1 & 0.186 & 0.339 \\
& 2 & 0.124 & 0.049 \\
& 3 & 0.259 & 0.117 \\
& 4 & 0.466 & 0.220 \\
& 5 & 0.881 & 0.525 \\
\hline
\end{tabular}

Table 2: Descriptive statistics of the absolute judgment errors in $\mathbf{m}$ for the main effects. 


\subsection{Discussion}

In general, our results indicate that higher distances between the real world object and the viewer decreases the judgment accuracy, in accordance to Swan et al. [10]. This phenomenon is well known since binocular disparity works best at near distances, its effectiveness decreases in the distance [3].

Moreover, our results show that the accuracy of depth judgments depends on the VSD and the distance between VSD and real world object. In more detail, small VSDs allow for a better judgment of small distances while high VSDs improve the depth judgment for far distances. However, just shield 1 allows for a better judgment for the smallest VSD. The correlation of judgment error and distance between VSD and shield position (Figure 9) explains this effect. This means that higher parallaxes decrease depth judgment.

Our study produced data that quantify the accuracy of depth judgments a human can achieve using a stereoscopic see-through display. This data provides a benchmark for the development of software and hardware solutions for stereoscopic see-through displays. Among the tested VSDs, we identify a VSD between 5 and $8 \mathrm{~m}$ as promising for the case of a stereo HUD as it allows quite accurate judgments for near as well as far real world objects. For example, the judgment error for a real world object $16 \mathrm{~m}$ away from the viewer is $73 \mathrm{~cm}$ while close objects at a distance of $2.4 \mathrm{~m}$ involve inaccuracies of $19 \mathrm{~cm}$ on average for a VSD of $5 \mathrm{~m}$.

\section{IMPLICATIONS}

We conducted two user studies to investigate the comfort zone and the accuracy of depth estimations by augmenting the real world with a 3D see-through display. The results of our studies let us derive the following guidelines for developing a stereo HUD:

Choosing an appropriate VSD: The comfort zone as well as the depth judgment accuracy depend on the chosen VSD, which depends on the use case. We found that for a stereo HUD a VSD between 5 and $8 \mathrm{~m}$ constitutes a good trade-off for comfortably displaying both driving information such as speed close to the driver and at the same time allows for a feasible augementation of real world objects at further positions.

Choose virtual object positions carefully: The use of one depth layer compared to the use of multiple depth layers has a significant effect on the comfort zone. While just one layer allows for comfortably covering a large depth range, more than one object displayed on different layers lowers this range. This means that situations requiring larger parallaxes, such as tagging an approaching obstacle, can hide other virtual layers to comfortably highlight the respective depth position.

Tagging objects via binocular disparity: Depth positions can be judged accurately depending on the distance to the real world object. Our results provide a reference in two ways: First, each application that augments reality by tagging requires a certain accuracy. Second, our results determine the requirements the picture generating unit and the optics of a 3D seethrough display have to fulfil. The quality of stereoscopic visualizations should not limit the user's depth perception.

\section{CONCLUSION}

In this work, we investigate the comfort zone and the accuracy of human depth perception regarding stereoscopic see-through displays. Our work is motivated by the vision of an automotive stereo HUD that allows for semantically structuring information via depth.
We conducted two user studies to assess parameters that influence the technical as well as the content design. We chose a highly controlled setup with a static real world surrounding that maximized the internal validity of our results. In particular, we are interested in an appropriate VSD and the corresponding comfort zone, as well as in the human accuracy of depth judgments using a stereoscopic see-through display. For a stereo HUD, the collected data recommend a VSD between 5 and $8 \mathrm{~m}$ which involves a comfort zone ranging approximately from 3 to $20 \mathrm{~m}$ and allows judgment accuracies up to $73 \mathrm{~cm}$ for a $16 \mathrm{~m}$ distant real world object. We believe our data set is applicable to other areas with different requirements, e.g., to cover different depth ranges as a stereo HUD.

Acknowledgements. The project on which this work is based is funded by the German Federal Ministry of Education and Research, funding code 13N12602. We thank the Fraunhofer Heinrich Hertz Institute for the software support.

\section{REFERENCES}

[1] N. Broy, F. Alt, S. Schneegass, N. Henze, and A. Schmidt. Perceiving layered information on 3D displays using binocular disparity. In Proc. PerDis '13. ACM, 2013.

[2] N. Broy, E. André, and A. Schmidt. Is stereoscopic 3d a better choice for information representation in the car? In Proc. AutomotiveUI'12, New York, NY, USA, 2012. ACM.

[3] J. Cutting. How the eye measures reality and virtual reality. Beh. Res. Meth., 29, 1997.

[4] D. M. Hoffman, A. R. Girshick, K. Akeley, and M. S. Banks. Vergence-accommodation conflicts hinder visual performance and cause visual fatigue. Jour. of Vis., 8(3), 2008.

[5] G. Jones, D. Lee, N. Holliman, and D. Ezra. Controlling perceived depth in stereoscopic images. In Stereoscopic Displays and Virtual Reality Systems VIII, 2001.

[6] M. Lambooij, M. Fortuin, I. Heynderickx, and W. IJsselsteijn. Visual discomfort and visual fatigue of stereoscopic displays: a review. Journal of Imaging Science, 2009.

[7] N. Milicic and T. Lindberg. Menu interaction in head-up displays. In Human Factors, Security and Safety, 2009.

[8] P. Ott. Optic design of head-up displays with freeform surfaces specified by nurbs. In Proc. SPIE, 2008.

[9] T. Shibata, J. Kim, D. M. Hoffman, and M. S. Banks. The zone of comfort: Predicting visual discomfort with stereo displays. Journal of Vision, 11(8), 2011.

[10] J. Swan, M. Livingston, H. Smallman, D. Brown, Y. Baillot, J. Gabbard, and D. Hix. A perceptual matching technique for depth judgments in optical, see-through augmented reality. In Virtual Reality Conference, 2006, pages 19-26, March 2006.

[11] M. Tönnis, V. Broy, and G. Klinker. A survey of challenges related to the design of $3 \mathrm{~d}$ user interfaces for car drivers. In Proc. 3DUI'06, pages 127-134. IEEE, 2006.

[12] M. Tönnis, L. Klein, and G. Klinker. Perception thresholds for augmented reality navigation schemes in large distances. In ISMAR'08, pages 189-190. IEEE, 2008.

[13] H. Urey, K. Chellappan, E. Erden, and P. Surman. State of the art in stereoscopic and autostereoscopic displays. Proceedings of the IEEE, 99(4):540-555, April 2011.

[14] F. Wientapper, H. Wuest, P. Rojtberg, and D. Fellner. A camera-based calibration for automotive augmented reality head-up-displays. In Proc. ISMAR'13. IEEE, 2013.

[15] S. P. Williams and R. V. Parrish. New computational control techniques and increased understanding for stereo 3-d displays. In Proc. SPIE, pages 73-82, 1990. 